\title{
18. The warp and weft: Synthesising our taxonomic tapestry
}

\author{
Marc F Oxenham and Alison M Behie
}

While this volume has been structured around three key themes, reflecting key research interests of Colin's over the years, what has clearly emerged is the enormous influence Colin has had, at a range of levels and degrees of pervasiveness, on the thinking of many people. Our title, Taxonomic Tapestries, evokes Colin's legacy in a number of ways with Kelley and Sussman's (2007) paper on academic genealogies being particularly relevant in this context. Kelley and Sussman explore a series of ancestor (academic supervisor) and descent (PhD student) lineages in order to document, for the most part, the academic intellectual history of primatology. Indeed, we can all engage in such genealogical detective work either to explore our own intellectual roots, or simply to locate famous ancestors. Both of us, for instance, can directly (in a unilineal fashion) trace our roots back to Hooton and his descendent (student) Washburn, while our respective ancestries diverge quite markedly after that. Colin only needs to go back a single generation to John Napier to locate his illustrious ancestor and perhaps chief intellectual influence, during his formative years, at least. Such formative periods are relevant to our tapestry of influences as we are all shaped to a greater of lesser degree by our subsequent histories and networks of colleagues and acquaintances. The contributions to this volume are clearly illustrative of this point.

In this final chapter we wish to briefly explore a range of additional ideas and questions that have emerged from contributor's approaches to the three meta-themes (the warp if you like) encompassed by this volume: behaviour and morphology; evolution; and conservation. While you, as readers, may have constructed your own framework upon which to engage with the various contributions presented here, we, the editors, have been particularly struck by the following (various strands forming the weft if you like): (1) ways of looking at the universe (incorporating ideas on taxonomy, speciation and evolution); (2) how one actually does taxonomy; (3) the causes of speciation; and (4) the value (and pitfalls) of the phylogenetic species concept (PSC). The main warp and emergent weft go to form a complex tapestry of tightly woven ideas and themes that illustrate the breadth of research interests and influence of Colin Groves. 


\section{Ways of looking at the universe: Species, taxonomy and evolution}

A rather disparate collection of approaches to this theme can arguably be said to characterise the entire set of contributions to this volume, and this is despite the quite specific set of themes authors were asked to address. Far from being a problem, the rich quarry of ideas and positions has been served. We have restricted our discussion of contributions to this emergent theme by selecting those that have a historical basis. Ulrich Welsh provides us with a biography of the German polymath Adolf Remane, who published some 300 papers, including a significant number on primate evolution and dental morphology. He presents the idea that Remane's interests resonate with those of Colin and in exploring some of the influences on research and methodological approaches of Remane, a range of prominent historical figures and themes were explored. The main point being that none of us work in an intellectual vacuum and many of the meta-ideas we cherish may in fact represent the repackaging of theories and approaches that have been circulating for centuries or are currently circulating elsewhere by scientists from a different, possibly unconnected, academic lineage.

Juliet Clutton-Brock provides a very useful perspective on how biologists have viewed their universe by reviewing aspects of the intellectual history of the naming of things, or more specifically, the naming of living organisms. Given the number of chapters dealing with the concept of species, and other taxonomic categories, in one way or another, this chapter provides a very solid context for these discussions (see below for Clutton-Brock's contribution to 'doing taxonomy'). The theme of the recycling of meta-ideas implicit in Juliet's work, is an issue explored in some detail, while in a highly specific sense, in Oxenham's chapter on Lamarck.

As noted, in some instances, ideas ascribed to one prominent scholar can be seen in the work of others that were somewhat less fortunate in terms of the success of their career and subsequent academic traction they received: Oxenham deals with the pre-Darwinian evolutionist Lamarck. Much of his chapter discusses what the author sees as Lamarck's actual views on the processes and mechanisms of evolutionary change, an important aspect of which prefigures some views usually ascribed to Darwin. Moreover, Lamarck's understanding of the nature of species is dealt with in some detail as well, as it was fundamental to his particular evolutionary scheme.

Historical precedents aside, Natasha Fijn provides a very important insight into how we conceptualise difference and similarity. Fijn looks at wild and domestic horses in Mongolia from the perspective of both scientific and cultural taxonomies. Colin is connected in as much as he has worked on horse taxonomy 
in the past (and published on it), and was the author's doctoral supervisor or academic ancestor. Indeed, Fijn states Colin is the world expert 'on the morphology and taxonomy of Equus przewalskii, or the takhi'. In trying to see past the domestic/wild dualism, she poses some interesting questions regarding the role of animal behaviour (and human-animal behavioural interactions) in taxonomic or classificatory determinations (see below for Fijn's contribution to 'doing taxonomy').

Perhaps the least intuitively obvious exploration of how taxonomies inform our understanding of biological and cultural processes comes from the pen of archaeologists Peter Hiscock and Chris Clarkson. They review models seeking to explain the processes involved in the production of what are often seen as various types of lithic artefacts in Mousterian (Neanderthal) assemblages. They assess two of these models in the context of a specific assemblage and argue that tool types, for this assemblage at least, are not simply a function of varying levels of flake retouch (curation) over time, but that different forms are arrested stages in a continuum. They suggest the initial lithic blank form plays a significant role in finished flake form or type. They go on to argue that different models may explain particular artefact assemblages and no one approach fits them all. They caution that simply seeing flake end stage morphology as a function of retouch intensity may lead one to misinterpret past (hominid) behaviour to a significant degree. There is no easy pathway to Neanderthal cognitive abilities and behaviour through lithic artefact form. There is an implicit link here to the nature of things, or in the context of this book, the nature of species. The transformational processes Hiscock and Clarkson discuss with reference to final forms (types of lithic artefact) have analogues in the biological world. Their argument that blank form predicates final form will stir reflections by biologists on canalisation and evolution.

\section{How does one actually do taxonomy?}

While not a main theme in this volume, one contribution deals with the nuts and bolts of taxonomic methodology and principles in a contribution that details the process of naming a new species of Sulawesi rat after Colin (Lenomys grovesi). And what better tribute to Colin?

A further two chapters deal with the operational aspects of taxonomy a little less directly. For instance, Juliet Clutton-Brock directly tackles the issue of defining, or correctly naming, domestic species. This particular approach is quite compatible with Colin's own definition of the Phylogenetic Species Concept. And, yes, we agree the dingoes should have their own name and Canis dingo is more than appropriate! Natasha Fijn's chapter (mentioned above under 
a different thematic heading), read in conjunction with Juliet Clutton-Brock's discussion of domestic species, provides an interesting discourse on the way we see, think about and categorise domestic animals and these two approaches are all the more interesting as they wrote their chapters independent of the content and approach of each other.

Finally, we wish to discuss Kees Rookmaaker's contribution here, even though it is perhaps more at home under the theme (see below) looking at the pros and cons of the PSC. Kees provides us with a history of rhino systematics which forms a solid basis to an understanding of the development of systematics in general. The paucity of recognised rhino species in Linneaus's time, to a slight increase in the time of Cuvier, sees an explosion in recognised forms when otherwise small differences (e.g. horn shape and temperament) were considered sufficient taxonomic signifiers. By the end of the nineteenth century a more considered approach to rhino taxonomy prevailed, a sort of compromise between extreme splitting and lumping as it were. The author then looks at Colin's rather substantive contributions to the taxonomy of rhinos, much in the form of his subscription to the PSC. Kees makes the same link back to the issue of conservation that other chapters have made, with the somewhat dour prediction of the possibility that taxonomy may end up serving the role of a way to study past biodiversity rather than to serve one of its key roles in helping protect current biodiversity. This idea is also alluded to in Angela Meder's chapter (discussed under the theme below) where she addresses the fact that 'while taxonomy is more exciting to study for living rather than extinct animals' the rapid decline of gorillas may not give us that luxury.

\section{Some causes of speciation}

A number of contributions explored, either implicitly or explicitly, mechanisms leading to speciation events, or contributing to morphological change or elevated levels of variation. For instance, Peter Andrews and Richard Johnson in reviewing the fossil evidence for gibbon ancestors find it somewhat lacking. They provide a thoroughly useful discussion of problems with actually identifying last common ancestors, which by definition cannot have homologous characters defining their descendent new species. With respect to the speciation theme Peter and Richard explore three scenarios, or potentially (future) testable hypotheses, for the origins of the hylobatids, but stress that however they came into being it was relatively recent (within the last few million years) and likely in the context of some severe environmental stressor.

This idea of the role of environmental stressors is also pursued by one of us, (AB) along with a number of colleagues, where the possible role of severe weather 
conditions as a variable in speciation events or, minimally, the biogeographic distribution of closely related species of primates (in this instance) is explored. It was found that group size was a key variable in adaptation to severe environments (i.e. frequent severe storms that destroy significant portions of the regular food supply). This, coupled with establishment of energy conservation behaviours by species faced with environmental stochasticity and the resultant stress (e.g. a hurricane wipes out the forest), provides a selective advantage in regions that are at high risk of extreme weather on a somewhat regular basis. Clearly, ecological niches can be characterised by extreme weather, as much as by any other variables.

Angela Meder's chapter focuses on the conservation of gorillas and the fact that due to habitat disturbance and hunting one of the major threats current gorilla populations deal with is small population size. She describes how many of these populations (as small as 10 animals in some instances) are isolated from each other, posing the threat that they may easily die off. However, another risk from this scenario is that they will potentially become further speciated from related species. While Angela notes that recent studies of the Cross River gorilla have shown populations that were once believed to be isolated are in fact exchanging genetic material, it is important to consider that these small, isolated populations could further change gorilla taxonomy.

While not a speciation event per se, David Bulbeck (a former graduate student of Colin's and later colleague) used human tooth size and shape analyses to explore the population history of Indo-Malaysia from the Late Pleistocene through to the modern period. He found a generalised trend in reducing tooth size, but no specific evidence for a reduction in tooth size from the Neolithic into the late Holocene. He favours a migration model, rather than an environmentally based one, whereby newcomers, perhaps originating in Sulawesi, have influenced the dental size and shape history of the region from the late Holocene to modern times.

Another former graduate student, and current colleague of Colin's, Debbie Argue, has also looked at speciation events, this time among a range of Early and Middle Pleistocene hominins. Argue has carried out a cladistic analysis of the Ceprano, Bodo, Daka and Kabwe fossils and presents data in support of placing these specimens into a common species: $H$. rhodesiensis. Colin had previously seen Bodo and Kabwe, at least, as distinct to H. erectus and preferred to see them as $H$. sapiens heidelbergensis. However, Debbie agrees with Colin's earlier work that these specimens, which she now sees as belonging to the same species, are quite distinct to $H$. erectus. She argues that the key evolutionary trait shared by members of this new species is a marked expansion in the cranial vault. Whether Colin would agree with these findings or not, he would be the 
first to applaud the approach which is, after all, in line with his version of the phylogenetic species concept. Standing on the shoulders of giants is perhaps something Debbie is quite proud of in this instance.

Before leaving the emergent theme of speciation, a further past graduate student of Colin's, and now colleague, Michael Westaway (and his associates) stress the importance of Colin's interpretation of the PSC and the resultant definition of more, rather than fewer, species. Minimally, splitting facilitates hypothesis testing as to the validity of such 'split' species, while the opposing approach (lumping) does not. Colin's influence in this regard can be seen in the expansion in the number of recognised hominin species in Pleistocene Southeast Asia, an early 'pre-erectine' species of which may be ancestral to Homo floresiensis. In terms of anatomically modern human variation in the region, the authors see microevolutionary change as key, perhaps in the context of environmental and climatic influences and not ruling out the possibility of more prosaic explanations; sexual dimorphism for instance. They see no role for previously held views of genetic inputs from Homo erectus, or similar such species. Notwithstanding, the new genetic data indicating Neandertal and Denosovan influences seems quite exciting.

\section{The value (and pitfalls) of the phylogenetic species concept (PSC)}

A number of contributors have dealt more or less explicitly with the PSC (with Kees Rookmaaker's chapter, while relevant in this regard, discussed under the theme of 'doing taxonomy' above). The nature of the species concept biologists ascribe to would seem to have important positive and negative ramifications for a range of sub-disciplines, not the least being conservation and parasitic disease control. Robert Attenborough, a long-time colleague of Colin's, explores Colin's version of the Phylogenetic Species Concept, which had led him to split numerous taxonomic units into smaller ones by identifying more species than other operational species concepts might allow. Robert develops an argument for the value of this approach, particularly where seeing more species may be of some practical benefit. He applies this idea to his review of the taxonomy of mosquitoes that have the capacity to infect humans with the malarial parasite and stresses the need to fine tune our taxonomy of these organisms if we are ever to make advances in interrupting the malarial cycle.

John Oates and Nelson Ting draw a detailed and well-argued direct link between the realities of institutionally funded conservation efforts and taxonomy. While 
they are quick to stress that taxonomy needs to be carried out independently of the direct needs of conservation priorities (in order to preserve the scientific rigour and independence of systematics), they stress that:

Species are the common primary 'currency' used in biodiversity conservation planning. Regions and ecosystems are often prioritised for conservation action based on measures of species richness and endemism and species judged to be in danger of extinction are usually given special attention ... [but] unstable classifications can have implications for conservation priorities and policies.

While the authors try and avoid taking sides in terms of subscription to one major approach (BSC vs PSC) over another, they tend to side with taxonomies that are a result of PSC approaches, in as much as the operational practicality of the PSC appears more objective, even if it does tend to lead to a proliferation of taxonomic units rather than a reduction. Notwithstanding, even subscribing to the PSC has its own problems. For instance, the authors note that with respect to red colobus monkey classification, even those who agree on subscribing to the PSC argue over which traits are important in classifying/differentiating this group. At the end of the day, the authors advocate consistency and consensus (and soon!) with regard to colobus monkey systematics, before a lack of such inadvertently leads to more extinction events (see Kees Rookmaaker's similar lament with respect to rhino taxonomy).

Eric Meijaard and Ben Rawson, both previous students of Colin, note the significant and far reaching impact Colin has had on both pig and gibbon taxonomy. Indeed, they argue that his work in this area has made a positive contribution to the conservation of both groups. In general, the authors address the issue of the effect on conservation efforts of greater or lesser taxonomic differentiation. For some Southeast Asian pigs, at least, better identification of endangered species (recognised using the PSC) has been a boon in terms of conservation success. The same can be argued for several species (recognised by Colin) of gibbon. However, interestingly, a survey of those involved in gibbon conservation efforts suggested that taxonomic classifications, per se, were not a driver, or detractor, with respect to conservation as conservation efforts tend to focus on endangered populations and not species or sub-species. One important point, in the context of the current heated discourse over taxonomic instability, is that the authors do not see a trend whereby conservation funds are diluted in the face of taxonomic inflation.

The main purpose of this edited volume was to pay tribute to the incredibly productive career of Colin Groves in the context of a complex tapestry of 
Taxonomic Tapestries

bio-evolutionary themes linked by the common glue of taxonomy. We believe we have been successful in this regard and as editors, are honoured to have the privilege of packaging this tribute to a remarkable scholar: Colin Groves.

\section{References}

Kelley E, Sussman RW. 2007. Academic genealogy on the history of American Field Primatology. Am J Phys Anthrop 132:406-425. 
This text is taken from Taxonomic Tapestries: The Threads of Evolutionary, Behavioural and Conservation Research, edited by Alison M Behie and Marc F Oxenham, published 2015 by ANU Press, The Australian National University, Canberra, Australia. 